\title{
Mechanism of idiosyncratic drug induced liver injury (DILI): unresolved basic issues
}

\author{
Rolf Teschke $^{1}$, Jack Uetrecht ${ }^{2}$ \\ ${ }^{1}$ Department of Internal Medicine II, Division of Gastroenterology and Hepatology, Klinikum Hanau, Hanau, Academic Teaching Hospital of the \\ Medical Faculty of the Goethe University Frankfurt/ Main, Frankfurt/Main, Germany; ${ }^{2}$ Department of Pharmaceutical Sciences, University of \\ Toronto, ON, Canada \\ Contributions: (I) Conception and design: Both authors; (II) Administrative support: None; (III) Provision of study materials or patients: None; \\ (IV) Collection and assembly of data: None; (V) Data analysis and interpretation: Both authors; (VI) Manuscript writing: Both authors; (VII) Final \\ approval of manuscript: Both authors. \\ Correspondence to: Rolf Teschke, MD. Professor of Medicine, Department of Internal Medicine II, Klinikum Hanau, Teaching Hospital of the Goethe \\ University of Frankfurt/Main, Leimenstrasse 20, D-63450 Hanau, Germany. Email: rolf.teschke@gmx.de.
}

\begin{abstract}
Clinical features of idiosyncratic drug induced liver injury (DILI) are well described in cases that have been assessed for causality using the Roussel Uclaf Causality Assessment Method (RUCAM), but our understanding of the mechanistic steps leading to injury is fragmentary. The difficulties describing mechanistic events can be traced back to the lack of an animal model of experimental idiosyncratic DILI that can mimic the genetic requirements of human idiosyncratic DILI. However, immune tolerance plays a dominant role in the immune response of the liver, and impairment of immune tolerance with immune checkpoint inhibitors increases DILI in both humans and animals. This may provide one method to study the individual steps involved. In general. the human DILI liver is a secret keeper providing little insight into what occurs in the diseased organ. Sufficient evidence exists that most idiosyncratic cases are mediated by the adaptive immune system, which depends on stimulation of the innate immune system, but the triggering factors are unknown. It is attractive to hypothesize that the gut microbiome plays a role; however, it is very difficult to study. Similarly, exosomes are likely to play an important role in communication between hepatic cells and the immune system, but there is a lack of data on blood exosomes in affected patients. Reactive metabolites are likely to play an important role. This is supported by the current analysis, which revealed an association between metabolism by cytochrome P450 and drugs most commonly involved in causing idiosyncratic DILI with causality verified by RUCAM. Circumstantial evidence suggests that reactive oxygen species (ROS) generated by cytochrome P450 could be responsible for the initial steps of injury, but details are unknown. In conclusion, most of the mechanistic steps leading to idiosyncratic DILI remain unclear.
\end{abstract}

Keywords: Drug induced liver injury (DILI); idiosyncratic DILI; Roussel Uclaf Causality Assessment Method (RUCAM); innate immune system; lipopolysaccharides (LPS); gut microbiome; cytochrome P450; reactive oxygen species (ROS); oxidative stress; mechanistic steps

Submitted May 19, 2020. Accepted for publication Jul 22, 2020.

doi: 10.21037/atm-2020-ubih-05

View this article at: http://dx.doi.org/10.21037/atm-2020-ubih-05

\section{Introduction}

A broad range of chemicals are injurious to the liver as evidenced by clinical and experimental studies, which commonly provide clues to mechanistic steps leading to injury. Chemicals of clinical interests include alcohol (15) and aliphatic halogenated hydrocarbons such as $\mathrm{CCl}_{4}$ (carbon tetrachloride) (6-11). In addition, heavy metals are found in excess amounts in the liver of patients with genetic liver diseases such as primary hemochromatosis with excess 
of iron $(12,13)$, or Wilson's disease with excess of copper $(14,15)$. Clinically important are also toxic liver diseases caused by herbs leading to herb induced liver Injury (HILI) (16-19), and drugs presenting as idiosyncratic drug induced liver injury (DILI) (17,20-24).

Idiosyncratic DILI is viewed as a complex and complicated human disease $(17,20-30)$. The 10 drugs most commonly implicated in DILI worldwide starting with the most common are amoxicillin-clavulanate, flucloxacillin, atorvastatin, disulfiram, diclofenac, simvastatin, carbamazepine, ibuprofen, erythromycin, and anabolic steroids as body building agents (22). There is also a compilation of 46,266 worldwide DILI cases with established causality (23). However, data on the exact number of potentially hepatotoxic drugs are not available from these reports $(22,23)$ or other publications (26). A recent review cited studies that estimated the number of drugs that cause DILI to range from 385 to 1,266 drugs (30), and other estimates from the United States came up with more than 1,000 drugs that cause DILI, although causality has not always been established clearly as explicitly mentioned (27). On the other hand, based on DILI cases contained in the LiverTox database, 353 drugs with the potential to injure the liver were recognized (25). The high variability of drug numbers is likely due to the lack of clear criteria of case inclusion or exclusion and confounding variables, poor case data quality, alternative causes, and incomplete causality assessment by a robust algorithm $(22,23,25-31)$. It seems that perhaps a few hundred drugs are potentially hepatotoxic, but the exact number remains uncertain.

There is also much uncertainty on initial mechanistic steps leading to idiosyncratic DILI. Although much attention has been paid to this topic worldwide, it is a challenging issue due to lack of appropriate animal models leaving many open questions (24). These scientific conditions remind us of the musical "The unanswered question" by the US Charles Ives.

The aim of this article is to analyze unresolved basic issues of mechanistic steps in idiosyncratic DILI. Special attention is given to immune aspects in connection with the gut microbiome and the hepatic cytochrome P450 (CYP). It is hoped that a pragmatic and conceptual approach can at least partially close the gaps in our understanding of the mechanistic steps occurring initially and during the cascade of injurious events.

\section{Literature search and source}

The PubMed database was searched for articles on DILI by using the following terms: drug induced liver injury, DILI, idiosyncratic DILI, RUCAM, pathogenesis, mechanistic steps, adaptive immune system, innate immune system, HLA genes, hepatic cytochrome P450, reactive oxygen species (ROS), exosomes, and gut microbiome, whereby search terms were used alone or in combination. The electronic search was completed on 15 April 2020 and supplemented by a manual literature search, using also the private archives of the authors. Preference was given to articles in the English language. The final compilation consisted of original papers, consensus reports, and review articles with the most relevant publications included in the reference list.

\section{Definition}

Idiosyncratic DILI is a special type of human liver injury that is specific to an individual and does not occur in most patients treated with a drug. It is also idiosyncratic in animals, which poses a major challenge for developing animal models. Human idiosyncratic DILI reflects both the properties of the drug, with its special physicochemical features, and its interaction with a susceptible individual based on genetic and other unknown factors $(23,24,32)$. In contrast, human intrinsic DILI is caused by specific toxic properties of the drug and occurs in virtually any human and most animals through mechanisms not requiring genetic predisposition $(23,32)$. Differentiation between human idiosyncratic and intrinsic DILI is essential if clinical and mechanistic aspects are to be considered to avoid descriptions of idiosyncratic DILI characteristics based on mixed results of both DILI forms. The present article focuses on unresolved mechanistic steps of idiosyncratic DILI without extrapolation of mechanistic principles known for intrinsic DILI. Whereas mechanistic steps for drugs causing intrinsic DILI are commonly based on clear experimental or clinical evidence, this condition is not the case for idiosyncratic DILI, which relies mostly on circumstantial evidence.

\section{Unresolved key questions of basic mechanistic issues}

There are a number of interesting facts related to drugs and idiosyncratic DILI that warrant further consideration (Table 1). Although most of the clinical features are well established, a variety of mechanistic steps remain unresolved in this complex disease. They include drugs with 
Table 1 Fact sheet on drugs and DILI and unresolved mechanistic issues

Facts of drugs and idiosyncratic DILI
Several hundred drugs are potentially hepatotoxic as
compared to only a few drugs that appear never to cause
idiosyncratic DILI

Although there are some common features, idiosyncratic DILI caused by different drugs is not uniform. In addition, the features of DILI overlap with liver injury caused by other agents such as viral hepatitis

The same drug may cause two or more different types of liver injury as assessed by liver histology and laboratory tests

Although the idiosyncratic DILI caused by some drugs is strongly associated with a specific HLA genotype, only a small fraction of patients with that genotype will experience DILI when exposed to the associated drug

Although in most cases it has never been tested, autoantibodies are found in the serum of patients with idiosyncratic DILI caused by some drugs

There is strong evidence that the immune system is responsible for idiosyncratic DILI caused by many drugs

Adverse reactions mediated by the adaptive immune system require a prior activation of the innate immune system

Some patients treated with potentially hepatotoxic drugs show clinical and laboratory signs of immunoallergy or even autoimmunity while others do not

Exosomes appear to be an essential mechanism by which organs such as the liver communicate with the immune system. For example, drug-modified proteins are present in the exosomes from drug-treated hepatocytes and are taken up by antigen presenting cells

Endotoxins such as lipopolysaccharides have been detected in patients with liver injury unrelated to the use of drugs, and it has been proposed that they are pathogenic. This has not been tested in patients with idiosyncratic DILI. Patients with inflammatory bowel disease do not appear to be at increased risk of idiosyncratic DILI

There is a lack of an animal model that has the human HLA genotype required to mimic the full picture of human idiosyncratic DILI
Unresolved questions of basic mechanistic issues

What are the differences between the two groups? Is it lipophilicity and active transport that concentrates the drug in the liver, reactive metabolite formation with the formation of neoantigens, interference with basic pathways such as BSEP inhibition leading to cell stress and the release of DAMPs, or a combination of such factors?

What is the cause for the inhomogeneity of liver injuries? Can they be explained by differences in the mechanistic steps, or is it just the usual interindividual differences in the immune response to various agents?

Hepatocellular injury is the most common type of injury as compared with cholestatic injury and the rarer autoimmune DILI. Is an important factor that some drugs/metabolites are concentrated in bile?

What other risk factors in addition to HLA genotype are required? Is it the T cell receptor repertoire and/or other factors such as the gut microbiome?

It is unclear whether such autoantibodies are pathogenetic or simply an indication of an immune response against the drug

Are there other mechanisms of idiosyncratic DILI caused by some drugs that do not involve the immune system?

What mediators are responsible for activation of the innate immune system? Are they produced solely by hepatocytes or are non-parenchymal cells also involved?

Do differences in the chemical structure of drugs determine whether they can induce an immune response, and is prediction of liver injury possible in a setting of drug development?

Can exosomes from patients with idiosyncratic DILI confirmed by RUCAM be used to more accurately differentiate DILI from other types of liver injury. Can a study of exosomes provide a better fundamental understanding of the mechanisms of idiosyncratic DILI

Do the presence of serum endotoxins indicate that they are important in the pathogenesis of liver injury, or are they simply an indication of decreased liver function with a failure of Kupffer cells to clear them?

Can the early innate immune response to drugs that cause idiosyncratic DILI be studied in humans, or even in animals, even though, without the required HLA/T cell receptor repertoire, it does not lead to significant liver injury. If so, this could provide a way to predict that a drug candidate would cause idiosyncratic DILI in some patients

Table 1 (continued) 
Table 1 (continued)

Facts of drugs and idiosyncratic DILI

There is a strong correlation between reactive metabolite formation and the risk that a drug will cause idiosyncratic DILI. However, it is very difficult to prove that a specific reactive metabolite is responsible for idiosyncratic DILI
Unresolved questions of basic mechanistic issues

If reactive metabolites are responsible for most idiosyncratic DILI, what role do they play: neoantigen formation, production of ROS, other cellular damage leading to the release of DAMPs, etc.? Do enzymes other than CYP such as glucuronosyltransferase also play an important role in metabolic activation leading to idiosyncratic DILI?

In addition to hepatocytes, various non-parenchymal cells of How can data derived from experimental studies, not from the liver of the liver and immune cells outside of the liver are considered patients with idiosyncratic DILI, be translated to human DILI in the absence to be involved in the pathogenesis of idiosyncratic DILI of the required HLA molecules?

Multiple hepatic mediators released from hepatocytes and The abundance of mediators and resulting hypotheses is challenging; non-parenchymal cells have been implicated to contribute to how valid is it to translate results from mostly in vitro studies to human idiosyncratic DILI development. idiosyncratic DILI?

There are abundant publications that propose various mechanisms of idiosyncratic DILI

There are multiple mechanistic studies, many in vitro and at high drug concentrations. Other studies involve drugs or chemicals that are intrinsically toxic

How can mechanistic hypotheses be rigorously tested?

Many of the results from these studies are not reliable indicators of the mechanism of DILI in humans. Mechanistic hypotheses must be consistent with the characteristics of idiosyncratic DILI in humans, and whenever possible, mechanisms should be tested in humans

BSEP, bile salt export pump; CYP, cytochrome P450; DAMPs, danger-associated molecular pattern molecules; DILI, drug-induced liver injury; HLA, human leucocyte antigen; ROS, reactive oxygen species; RUCAM, Roussel Uclaf Causality Assessment Method.

variable chemical structures, variabilities of clinical features and genetics of patients at risk, the multiplicity of non parenchymal cells in addition to the hepatocytes exposed to the drugs entering the liver following intestinal absorption, and multiple immune cell types.

\section{The human study model of idiosyncratic DILI}

Idiosyncratic DILI mechanistic steps are best analyzed using patients with their unique genetic profile and other unknown risk factors instead of experimental models that lack these features. Inclusion in study cohorts will require patients with the diagnosis of real idiosyncratic DILI verified by a robust causality assessment method (CAM) such as the Roussel Uclaf Causality Assessment method (RUCAM), established in 1993 (33) and now the preferred version updated in 2016 (34). This will ensure cohort homogeneity through exclusion a priori cases with alternative causes unrelated to drug use. Indeed, it was early recognized that up to $47 \%$ patients with the initial diagnosis of idiosyncratic DILI had been wrongly diagnosed in face of overt alternative causes (35), findings in principle, confirmed in subsequent reports $(28,29)$.

A prerequisite for evaluating mechanistic steps is the use of patients with high RUCAM based causality gradings of probable or highly probable. At the present time RUCAM cannot be replaced by any of the multiple diagnostic biomarkers that have been heavily promoted by interested parties, because virtually all biomarkers available on the market came under scientific fire recently due to misconducted studies carried out by groups outside the European Medicines Agency (EMA) (36,37). In more detail, EMA provided a statement on 17 April 2019 via internet that the earlier issued "Letter of Supprt for druginduced liver injury (DILI) biomarker (EMA/4238702016) had been retracted from the EMA website on 15 April 2019. The decision was based on information received by the IMI TransBioLine consortium, which is a successor of the former SAFE-T consortium, which itself was the applicant of the "Letter of Support" issued in 2016. The consortium indicated that, due to a case of scientific misconduct at one of the collaboration partner centers of the consortium, the IMI TransBioLine consortium is no longer recommending the exploratory use of hyperacetylated HMBGB1 isoforms in clinical studies. The overall promising nature of other recommended biomarkers was considered to be highly dependent on the results for the incriminated biomarker HMGB1. The CHMP/EMA has therefore decided to retract this Letter of Support that affected many other biomarkers that had 
been initially supported (37). A detailed discussion on this subject was published earlier (36).

\section{DILI liver as a secret keeper organ}

The liver of patients with idiosyncratic DILI is not readily accessible for investigations exploring the initial steps triggering the development of liver injury. In particular, invasive diagnostic procedures such as liver biopsies are not practicable and must be declined for ethical reasons considering the lack of any significant benefit to the patient. Liver histology is also not part of the diagnostic algorithms of RUCAM (34). Instead, we must rely upon biomarkers released into body fluids, usually blood.

\section{Serum enzyme activities and bile acids}

In patients with idiosyncratic DILI, a battery of laboratory parameters, including aminotransferases, merely signify the existence of a liver disease (37). More specifically, increased serum activities of glutamate dehydrogenase (GDH) would provide circumstantial evidence of an involvement of mitochondria in liver injury, whereas increased serum bile acids (BA) would reflect drug associated injury of the biliary system (37). However, these two parameters do not necessarily allow any conclusions on the underlying mechanistic steps leading to injury. These, and other laboratory parameters, indicate different types of liver injury: one is for hepatocellular injury and the other one for cholestatic injury (34). It is obvious that with at least 2 different liver injury types, a uniform hypothesis based on a single mechanistic step may not be feasible. Also in face of the variability of liver histology that includes, for instance, steatosis, granulomatous hepatitis, vanishing bile duct syndrome, and hepatic sinusoidal obstruction syndrome (HSOS) (38), one single mechanism may not be able to explain the liver histology of idiosyncratic DILI. However, if idiosyncratic DILI is mediated by the adaptive immune system, it is common for different individuals to have quite different responses. For example, the response to SARS$\mathrm{CoV}-2$ and the manifestations of the infection varies widely between different individuals. Some individuals become quite ill including a "cytokine storm", while others have little or no symptoms; some individuals lose the sense of smell while others do not, some have gastrointestinal symptoms or strokes while others do not. Such variations in individual responses are common, especially when the immune system is involved.

\section{Blood exosomes}

Exosomes found in the blood of patients with various liver diseases have provided an important development in the study of liver injury. It has been proposed that they have the potential to serve as diagnostic markers for specific liver diseases $(39,40)$. In addition, they likely play an important mechanistic role in communication between the liver and the immune system. They have been studied both in patients and in animal models of liver injury (39-50). Belonging to the group of extracellular vehicles comprising also microvesicles and apoptotic bodies (40), exosomes represent vesicles, which are commonly derived from cell membranes of various organs including the liver (39). Exosomes released from the liver may be traced back to hepatocytes, hepatic stellate cells, and immune cells in normal and pathological conditions $(39,48)$. They carry lipids, proteins, coding and non-coding RNAs, and mitochondrial DNA (48). Most important for the mechanism of immune adaptation and immune idiosyncratic DILI, exosomes carry drugmodified proteins that are taken up by antigen presenting cells (48). In these studies, however, the exosomes did not lead to activation of the antigen presenting cells; therefore, in these cases immune tolerance may prevail rather than immune reactions associated with idiosyncratic DILI. In other studies, blood exosomes were detected in animals with liver injury caused by $\mathrm{CCl}_{4}(50)$, but they have not yet been studied and confirmed using the recently published animal model of $\mathrm{CCl}_{4}$ liver injury (7). In addition, they have not been examined in patients with liver injury following intoxication by $\mathrm{CCl}_{4}(8,9)$. Exosomes were also found in the blood of patients with alcoholic liver disease (ALD) and in animals with experimental alcoholic liver injury $(4,41,42)$. These blood exosomes contain CYP 2E1 originating from the injured liver where this CYP isoform is part of the hepatic microsomal ethanol oxidizing system (MEOS), which contributes to ethanol metabolism and the associated liver injury $(1,2,4,51)$. Increased exosomal CYP 2E1 is considered as a possible diagnostic biomarker in the blood of humans with alcoholism and microsomal stress. These exosomes may help disclose mechanistic steps leading to alcoholic liver injury, and they could assign alcohol as a cause in a liver disease of unknown etiology $(4,42)$. Exosomes are also released into the blood from the of liver of mice treated with acetaminophen (chemically known as $\mathrm{N}$-acetyl-p-aminophenol; APAP) leading to experimental intrinsic DILI $(41,42,45)$. However, their diagnostic or mechanistic role in human intrinsic HILI by APAP remains 
to be established $(42,44,45,49)$. Surprisingly, no data on blood exosomes in patients with idiosyncratic DILI have been published (38-40,42-50). Instead, experimental studies only showed that exosomes originating from hepatocytes are closely related to exosomes derived from cholangiocytes that can affect various signaling pathways (47). Whether these connections explain cholestatic injury in addition to hepatocellular injury in human idiosyncratic DILI remains to be established. Future studies may be useful for elucidating initial mechanistic steps of liver injury $(39,40,42-50)$.

\section{Blood microRNA}

MicroRNAs originating mainly from the liver are found in the blood of patients with liver diseases, either included in exosomes or freely floating $(41,52)$. They were promoted not only as diagnostic biomarkers (27,52-60), but also as mechanistic biomarkers providing insights into to pathogenetic steps in liver injury $(61,62)$. However, the enthusiasm for their use as diagnostic biomarkers was not shared by others because they lack superiority over existing parameters (63). In addition, there were methodology shortcomings (64). In particular, the combination of diagnostic biomarker qualities and idiosyncratic DILI is problematic because many biomarkers were not validated using cases of idiosyncratic DILI assessed for causality by RUCAM with causality gradings limited to highly probable or probable ones $(36,64)$. In addition, most biomarkers have been described for human or animal intrinsic DILI caused by APAP overdoses, providing results that are not necessarily suitable for translation to human idiosyncratic DILI (27,36,52-61). Of note, blood microRNA was analyzed in capillary blood of RUCAM based cases with intrinsic DILI caused by APAP and in one single case associated with the use of clarithromycin causing idiosyncratic DILI (52), but no studies were carried out in patients with idiosyncratic DILI by drugs others than clarithromycin (61). Respective data were also not published for the 46,266 cases of RUCAM based idiosyncratic DILI (23). To date, it appears that microRNA analyses have failed as a diagnostic biomarker of idiosyncratic DILI.

With respect to microRNA, other problems emerged $(23,36)$. Triggered by promotional statements issued by regulators and consortia, a scientific and clinical biomarker hype emerged in 2016 following EMA's online presentation of a Letter of Support to use several diagnostic biomarkers including microRNA in order to verify or exclude liver injury cases as summarized recently (36). However, on 15 April 2019, confusion emerged due to the EMA issuing a retraction note regarding microRNA and various other potential biomarkers due to external data manipulation. This led to a dramatic scientific and regulatory dilemma because previously published analyses and recommendations now require reconsideration. The website with the retraction notification remained accessible only for a short period before it was removed from the internet. The initial letter of support was also removed. As a consequence, related regulatory or consortia Letters of Recommendations previously provided by the FDA and SAFE-T (Safer and Faster Evidence-based Translation) Consortium also disappeared from their websites. The lack of accessibility of the retraction notices likely explains why some reports on microRNA, other biomarkers, and DILI published after April 2019 did not consider this new state of affairs. It is also obvious that data derived from blood microRNA cannot be used to identify the multiple mechanistic steps in idiosyncratic DILI.

\section{Gut microbiome and blood lipopolysaccharides (LPS)}

There is a significant immunological interaction between the gut and the liver (65). For instance, evidence exists that the gut microbiome plays an important role in several types of liver injury. In particular, the presence of specific gut bacteria is associated with a decrease in the autoimmune reactions caused by immune checkpoint inhibitors used to treat cancer (66). There is also evidence that endotoxins, such as LPS derived from the gut microbiome, enter the hepatic blood vessels through a leaky gut, and if not cleared by the liver, reach the systemic circulation where they can be quantified (66-69). They are under consideration as toxins for a variety of human and experimental liver injuries $(2,4,24,68,70-72)$ caused, for instance, by alcohol $(2,4,72)$, $\mathrm{CCl}_{4}(68,70)$, or APAP overdoses leading to intrinsic DILI (71). No published data on blood LPS are available in patients with idiosyncratic DILI when reports up to 2014 were evaluated (56). Findings of missing LPS data were currently confirmed using the PubMed database for search of respective reports published until mid of April 2020. Seemingly a neglected topic, the lack of LPS data requires further investigations in patients with idiosyncratic DILI with high causality gradings based on the use of RUCAM. As it presently stands, LPS cannot assist in clarifying mechanistic steps in human idiosyncratic DILI. In particular, the 
incidence of idiosyncratic DILI does not appear to be higher in patients with inflammatory bowel disease even though the liver in such patients is exposed to much higher levels of LPS and other inflammatory molecules.

\section{Blood monocytes}

Macrophages and monocytes play a critical role in the control of immune responses. Therefore, it seems plausible that they would play an important role in the mechanism of idiosyncratic DILI. Studies have shown that they even play a role in acetaminophen-induced DILI (73). In one study, peripheral blood monocytes from patients suspected to have diclofenac-induced DILI were cultured under conditions to convert them to "hepatocyte-like" (MH) cells (74). These cells increased the expression of Integrin beta 3 (ITGB3) when incubated with diclofenac. Cases were partially assessed for causality by the updated RUCAM (34), but some methodology uncertainties remained $(23,36)$. Integrin beta 3 (ITGB3) derived from $\mathrm{MH}$ cells was also promoted as a biomarker candidate for idiosyncratic DILI (34), but requiring conformation by peers as outlined previously $(23,36)$. Studies on MH cells and ITGB3 were published in 2018 based on Letters of Recommendations presented by EMA through IMI projects SAFE-T and MIP-DILI and supported by the US FDA (34). However, a few months later these parties revoked their recommendations in 2019 (36). In addition, the relationship between $\mathrm{MH}$ cells and hepatocytes as well as the steps leading to the production of MH cells are seemingly vague (74). At the present time, it is not possible to be confident of the relationship between ITGB3 produced by blood-derived $\mathrm{MH}$ cells and the mechanism of idiosyncratic DILI.

\section{Hepatic immune system}

Compelling evidence exists that for most idiosyncratic DILI cases the hepatic immune system plays a prominent pathogenetic role (24,56,74-77). A review of this evidence is beyond the scope of this paper, but is presented in a recent review (24). In short, a consensus exists that the hepatic immune system is involved in DILI caused by many drugs (24,56,74-77). Although there are multiple lines of evidence for an immune mechanism of most IDILI, and clinical evidence for alternative mechanisms is scant, given that biological systems are very complex, it is possible that some IDILI involves nonimmune mechanisms. In most cases the injury appears to be mediated by CD8 $\mathrm{T}$ cells of the adaptive immune system, which requires prior activation of the innate immune system. Early steps in this process likely involves activation of antigen presenting cells by molecules such as danger associated molecular pattern molecules (DAMPs) (24). Support for an involvement of the immune system in idiosyncratic DILI is provided by autoimmune parameters in the blood of patients (20) and clinical features, liver histology, and in some cases with human leucocyte antigen (HLA) genotypes (24).

An association of HLA genes as risk factor for idiosyncratic DILI caused by selected drugs has been described in a variety of reports $(17,20,21,24)$, but their clinical utility for use as a pre-prescription screening tool with potential liver injury remains unclear (24). Pharmacogenetic HLA associations were found in a few, but not all, patients under a treatment with a few drugs including abacavir, amoxicillin clavulanate, flucloxacillin, isoniazid and other antituberculosis drugs, lumiracoxib (21), and other drugs (20). In addition, for many published DILI cases, an association with HLA genes has been assumed, but it is unclear whether the liver injury was induced by drugs or due to alternative causes $(17,21-23,25,26,28,29,35)$ not carefully excluded by the use of a strong causality assessment tool such as the updated RUCAM (34). Concern also exists that for most drugs, there are too few cases available for investigators to evaluate (24). Although it is likely that most idiosyncratic DILI is immune mediated, the details of how a drug induces an immune response leading to DILI are unknown and likely different for different drugs $(24,78)$. Among the possible general mechanisms are that reactive drugs such as $\beta$-lactams or reactive metabolites can act as haptens to form neoantigens, and they can also cause cell damage leading to the release of DAMPs and activate inflammasomes. There is a large amount of circumstantial evidence for the involvement of reactive metabolites in the mechanism of idiosyncratic DILI; however, it is very difficult to prove that a specific reactive metabolite is responsible for human idiosyncratic DILI caused by a specific drug. Drug metabolism occurs in the liver preferentially via CYP $(79,80)$ and to a lesser extent through non CYP pathways (81). In particular, aryl glucuronides have been proposed to be responsible for DILI caused by carboxylic acids such as diclofenac; however, the evidence is far from compelling (82).

\section{Hepatic cytochrome P450 and oxidative stress}

CYP is primarily localized in the liver and degrades many 
drugs to harmless metabolites; however, it also has the potential to produce toxic metabolites $(79,80)$, which have the potential to initiate idiosyncratic DILI. To further evaluate this issue, top drugs causing idiosyncratic DILI as assessed in 48 cases worldwide with verified causality by RUCAM were analyzed for their possible metabolism via (83-100) CYP (101-118) (Table 2). In at least 28/48 drugs (58.3\%), clinical or experimental evidence exists that metabolism proceeds via CYP, whereas for the remaining 20 drugs $(41.7 \%)$ there were negative or missing results implicating CYP in the metabolism of these drugs (Table 2).

The CYP dependent metabolism of drugs and other

Table 2 Drugs causing idiosyncratic DILI with published causality assessment of the cases using RUCAM

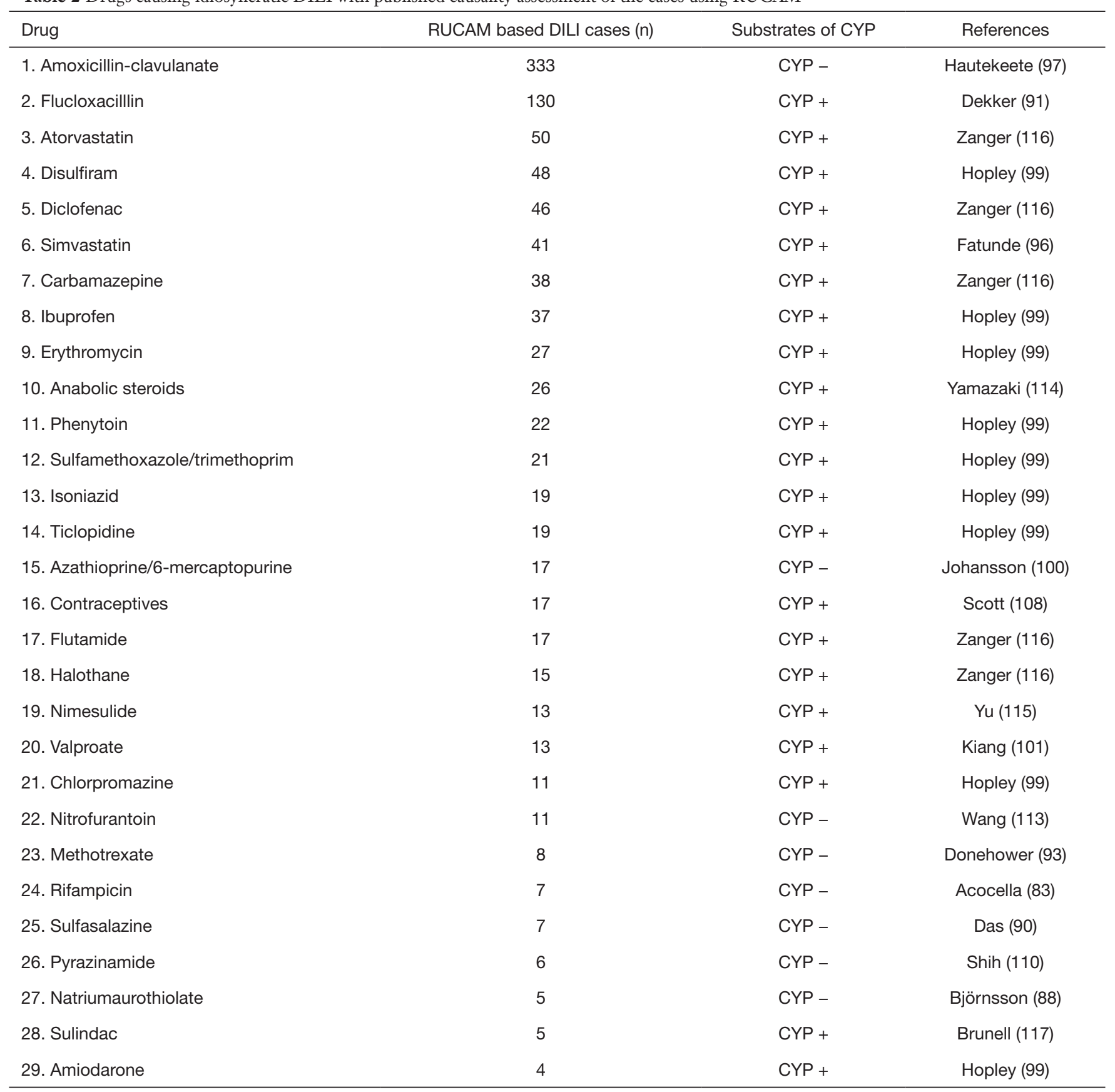

Table 2 (continued) 
Table 2 (continued)

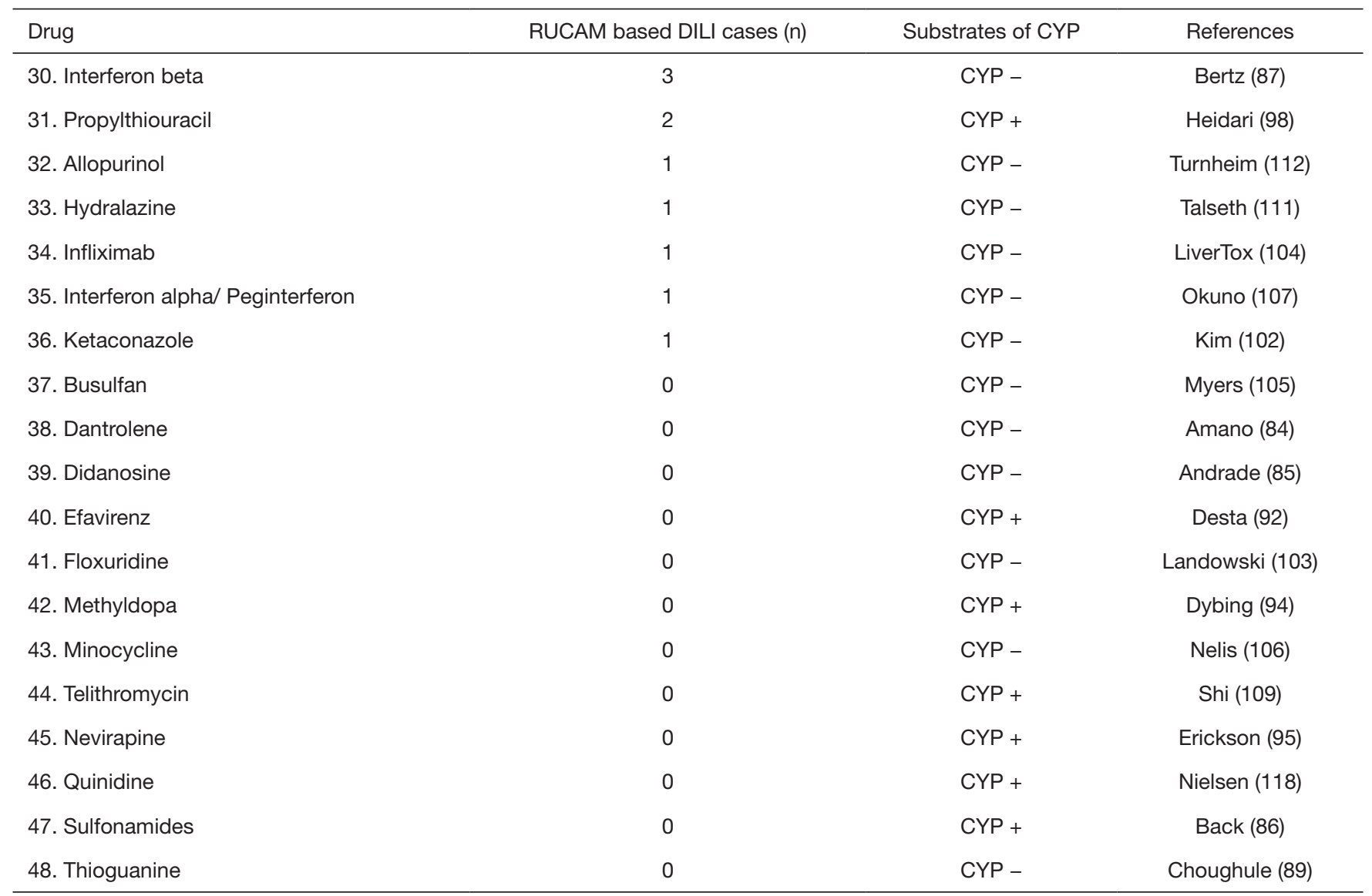

Listed are the top ranking 48 drugs worldwide causing idiosyncratic DILI with verified causality using RUCAM, with details presented in a previous publication (22). The references refer to the first author of the study that delineates whether the drug under consideration is a substrate of and metabolized by CYP (CYP +) or not (CYP -). CYP, cytochrome P450; DILI, drug induced liver injury; RUCAM, Roussel Uclaf Causality Assessment Method.

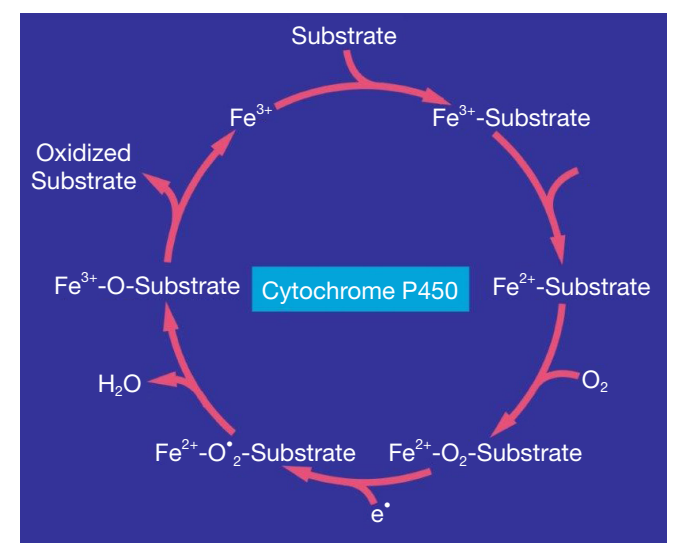

Figure 1 Metabolism of drugs and other substrates through the cytochrome $\mathrm{P} 450$ cycle. The figure is derived from a previous report (4). exogenous chemicals such as ethanol and $\mathrm{CCl}_{4}$ proceeds in the endoplasmic reticulum of the hepatocyte, which corresponds to the microsomal fraction obtained from liver homogenate after subcellular fractionation using ultracentrifugation (51). CYP is not a single enzyme but consists of several highly polymorphic isoforms (100). The individual steps of the catalytic CYP cycle are complex (119), presented in Figure 1 as a simplified multistep process (4). The reactions within the CYP cycle start with the binding of a drug as substrate to the ferric heme moiety of CYP$\mathrm{Fe}^{3+}$ (Figure 1). This is followed by the uptake of an electron provided by the NADPH-cytochrome $\mathrm{P} 450$ reductase that converts the ferric state of $\mathrm{CYP}-\mathrm{Fe}^{3+}$ to its ferrous state CYP-Fe ${ }^{2+}$, with subsequent inclusion of molecular oxygen and another electron provided again by the reductase (Figure 1) $(4,119)$. These reactions are supported by 
phospholipids, which are essential components of the microsomal membrane (4), and could be involved as peroxidized phospholipids in the development of the complex consisting of the reduced CYP, an activated form of oxygen, and the substrate (Figure 1). Under conditions of incomplete oxygen splitting, radicals may be generated from oxygen, phospholipids, and drugs, but the individual mechanistic steps occurring in the human liver of patients with idiosyncratic DILI are difficult to assess and are currently unknown. Finally, and under physiological conditions of drug use, the drug is split off as oxidized substrate, whereas the CYP molecule returns from its ferrous state $\left(2^{+}\right)$to its ferric state $\left(3^{+}\right)$and is again available for binding to the next substrate molecule to keep the CYP cycle running (Figure 1). Clearly, CYP presents with 2 sides of a coin: on the one side, CYP metabolizes drugs and clears them from the body, but on the other side, it produces ROS that may attack membrane structures of cell organelles. The damage produced could lead to direct liver injury, although it is harder to explain why this would be idiosyncratic, and it could also cause the release of DAMPs that promote immune mediated injury in susceptible individuals $(24,56,75-77,120,121)$. The reactive species could also act as haptens that provoke an immune response in susceptible individuals (120).

In addition to CYP in the endoplasmic reticulum, hepatic mitochondria also contain CYP, which possibly contributes to mitochondrial injury as suggested by experiments in alcohol-induced liver injury (4). Furthermore, hepatocytes release exosomes containing CYP as a result of toxicity associated with drug metabolism $(122,123)$, which is analogous to exosomal CYP found in the blood of patients with ALD or animals with alcoholic liver injury (42). However, data from studies of exosomes in the blood of patients with idiosyncratic DILI containing CYP are not available that could be used to substantiate a mechanistic role of CYP in this disease (43). In idiosyncratic DILI caused by a few drugs, anti-CYP can be detected in the blood, implying a close association between the incriminated drug and CYP (124-126) in line with the proposed involvement of the adaptive immune system (24). Many questions relating to an association between CYP polymorphisms and the risk of idiosyncratic DILI remain unanswered $(24,127)$.

Cellular oxidative stress in idiosyncratic DILI includes mitochondrial stress $(128,129)$ and microsomal stress $(129,130)$, and this may be related to ROS generated by CYP in these subcellular domains of the hepatocytes (128-
130), but specific ROS types involved in human idiosyncratic DILI have not yet been identified. For alcoholic liver injury, which involves the MEOS and is dependent on CYP and NADPH-CYP reductase, the following radicals have been implicated: singlet radical ${ }^{1} \mathrm{O}_{2}$, superoxide radical $\mathrm{HO}_{2}{ }_{2}$, hydrogen peroxide $\mathrm{H}_{2} \mathrm{O}_{2}$, hydroxyl radical $\mathrm{HO}^{\circ}$, alkoxyl radical RO', and peroxyl radical ROO' (4). Involvement of these radical types in the mechanism of idiosyncratic DILI is possible; however, despite multiple efforts, a satisfactory unifying mechanistic concept for idiosyncratic DILI with the involvement of various ROS types is currently not available.

\section{Hepatic non CYPs}

There are some drugs that cause idiosyncratic DILI validated by RUCAM, which do not appear to be metabolized by CYP (Table 2). In some cases, such as ß-lactams and busulfan it is likely because the drug is intrinsically reactive. In other cases such as methotrexate, the parent drug is toxic. In some cases, such as nitrofurantoin and dantrolene, reactive metabolites are formed by reduction rather than oxidation. In still other cases other enzymes may oxidize the drug, and it is unclear whether the generation of ROS may be involved $(81,131)$. Among these enzymes are aldehyde oxidase present in the cytosol, carboxylesterase, and UDP-glucuronosyltransferase (131). Despite FDA guidance documents that specify that acyl glucuronides are toxic (132), clear evidence that they are involved in the mechanism of idiosyncratic DILI is lacking. It will be very interesting to find out whether new chemical entities in the drug pipeline that are not metabolized by CYP are associated with a significant risk of idiosyncratic DILI. In particular, there are many new biological drugs that cause idiosyncratic DILI, and the mechanism presumably does not involve metabolism by CYP (133).

\section{Animal models}

Given the unpredictable nature of idiosyncratic DILI, it is impossible to prospectively study patients who will be affected. Animal models are very important for most biomedical research; however, idiosyncratic DILI is also idiosyncratic in animals. Therefore, it is difficult to develop animal models in which the incidence is sufficient to be practical for research. Most animal models involve high doses of the drug and/or other manipulations that lead to direct and immediate liver injury, and in general the 
histology is different $(24,134,135)$. These characteristics are different from the characteristics of idiosyncratic DILI; therefore, the mechanisms are almost surely different. Animals do not have the same HLA genotypes as those associated with an increased risk of DILI associated with specific drugs in humans; therefore, it would be impossible to develop an animal model to drugs that require a specific HLA genotype. However, not all idiosyncratic DILI appears to be associated with specific HLA genotypes. This is likely because some drugs react with so many endogenous proteins that there is likely one modified protein, which produces peptides. They in turn may bind to one of the HLA molecules that any individual human or animal may express.

A major development in the treatment of cancer has been drugs, which target immune checkpoints that prevent the immune system from destroying cancers (136). Two important immune checkpoints are programmed cell death protein 1 (PD-1) and cytotoxic T-lymphocyte-associated protein 4 (CTLA-4). The dominant immune response in the liver is immune tolerance. One side effect of these immune checkpoint inhibitors is DILI, and they also increase the risk of idiosyncratic DILI of coadministered drugs (137). The immune response is always a balance between an active immune response and immune tolerance, and immune checkpoint inhibitors shift this balance. Treatment of PD- $1^{-1-}$ mice with a combination of anti-CTLA-4 and amodiaquine (a drug associated with a relatively high incidence of idiosyncratic DILI) leads to a delayed onset liver injury with histology indistinguishable from that of idiosyncratic DILI in humans (138). This injury is mediated by CD8 T cells (139). Although the injury is significant and leads to decreased liver function with an increase in bilirubin, it does not lead to overt liver failure. This model also unmasks the potential of other drugs to cause DILI; however, the liver injury is less than with amodiaquine (140). It is likely that the lack of liver failure is due to the lack of the optimal HLA and T cell receptors required to produce the maximal amount of liver injury. In fact, drugs that cause serious liver injury are always associated with a higher incidence of mild liver injury, which often resolves with continued drug treatment. It is likely that most humans and even many animals have an innate immune response to drugs that can cause idiosyncratic DILI, but without the required HLA and T cell receptors, no adaptive immune response leading to injury occurs. A study of the innate immune response to drugs could provide a method to test the early immune events in idiosyncratic DILI and even a mechanism to screen drug candidates for the risk that they will be associated with a significant risk of DILI (24).

\section{Non parenchymal cells and mediators}

Given that the major site of reactive metabolite formation is hepatocytes, they are an important target of idiosyncratic DILI. However, there is a large amount of communication between hepatocytes and other cells in the liver, including Kupffer cells, hepatic stellate cells, and liver sinusoidal endothelial cells. In addition, immune cells in the liver and outside of the liver presumably also play important roles in the mechanism of idiosyncratic DILI. Injury to the liver usually causes a decrease in the number of Kupffer cells, but an increase in infiltrating monocyte-derived macrophages, which display a large amount of heterogeneity and change as the injury evolves (78). The number of publications on various pathogenetic aspects in idiosyncratic DILI is impressive $(17,21,24,32,61,75-77,121,127,128,130)$. The number of various hypotheses is not surprising given the variability of clinical features and the difficulty in performing rigorous mechanistic studies in humans. Therefore, it requires extrapolation from other types of experiments such as in vitro studies, studies with intrinsically toxic agents, and experiments using extreme conditions. In line with these considerations and restrictions, the conclusion can be reached that a satisfactory evidence for a unifying mechanism for individual susceptibility, initiation, and progression of idiosyncratic DILI is not available.

\section{Hepatocellular injury versus cholestatic injury}

The majority of idiosyncratic DILI presents clinically as hepatocellular injury; cholestatic injury is less common (19). This is why most studies have focused on the hepatocellular type rather the on the cholestatic injury (24). Circumstantial evidence suggests that the hepatocellular injury with its increased serum activities of ALT, AST, and GDH can be attributed to a variety of mechanistic processes that include immune and non immune systems, CYP and non CYP pathways, and mitochondria and non mitochondria targets of the hepatocytes. Conversely, pathogenetic steps leading to the impairment of the bile salt export pump (BSEP) of the hepatocyte as a cause of the cholestatic injury is evidenced by increased serum alkaline phosphatase (ALP) activities. Increased serum bile acid levels is less studied, but some details warrant mentioning (24). In particular, the composition of bile acids is different in rodents than 
in humans with a higher percentage of the more polar and less toxic taurine conjugates in mice and more of the toxic glycine conjugates in humans $(3,24)$. This complicates the development of a valid rodent model to study BSEP inhibition or other bile acid transporters, and it makes it more difficult to test the hypothesis that BSEP inhibition leads to cholestatic idiosyncratic DILI (24). Thus it remains to be established which mechanism triggers the cholestatic injury. However, it is likely that transport and concentration of a drug or its metabolites in the biliary system plays an important role.

\section{Conclusions}

Idiosyncratic DILI is a multifaceted disease that provides a large opportunity to resolve basic mechanistic issues. Clinical features are well described in cases assessed for causality using RUCAM, but a large portion of mechanistic steps triggering the liver injury remain unclear in face of both the behavior of the human DILI liver as a secret keeper organ and the lack of an appropriate animal model with genetic specificities of susceptible patients with idiosyncratic DILI. Sufficient evidence exist that the hepatic adaptive immune system mediates most of the liver injury cases, a process requiring stimulation of the innate immune system and transition to an adaptive response, but the events facilitating the stimulation remain unclear. Additional gaps of understanding relate to the failure to detect in the blood of patients with idiosyncratic DILI specific exosomes released from the injured liver that may carry information on coding and non-coding RNAs, mitochondrial DNA, and CYP isoforms that might help clarify initial mechanistic steps of injury. Data on patients' exosomes have not been published, either because they were not analyzed, or the levels were too low to provide a clear picture. Similarly, patients' blood microRNA has not been helpful to clarify existing mechanistic issues, and at present they represent a now outdated biomarker after losing regulatory support by EMA and the FDA following detected misconduct of an expert team. The current analysis also revealed that most of the drugs causing liver injury are metabolized by cytochrome P450 and NADPH cytochrome P450 reductase. These microsomal constituents may generate toxic ROS as by products with the potential to initiate liver injury, but evidence is only circumstantial. Given that drugs can target all non parenchymal cells such as Kupffer cells, hepatic stellate cells, liver sinusoidal endothelial cells, as well as intrahepatic granulocytes, lymphocytes, and monocytes, this makes it more difficult to define their role and the impact of active mediators for signaling and mechanistic pathways in human idiosyncratic DILI. In essence, from a scientific point of view, it would be desirable to elucidate the cascade of events with identification of each mechanistic step. A better understanding of the mechanisms of idiosyncratic DILI could lead to ways to prevent or treat it. However, at the present time and for the sake of patients, it is important to recognize early symptoms of idiosyncratic DILI and to quickly establish the diagnosis using a robust method for causality assessment such as the updated RUCAM assessment with the aim to discontinue the use of the incriminated drug.

\section{Acknowledgments}

Funding: None.

\section{Footnote}

Provenance and Peer Review: This article was commissioned by the Guest Editors (Ralf Weiskirchen and Wolfgang Stremmel) for the series "Unresolved Basic Issues in Hepatology" published in Annals of Translational Medicine. The article was sent for external peer review organized by the Guest Editors and the editorial office.

Peer Review File: Available at http://dx.doi.org/10.21037/ atm-2020-ubih-05

Conflicts of Interest: Both authors have completed the ICMJE uniform disclosure form (available at http://dx.doi. org/10.21037/atm-2020-ubih-05). The series "Unresolved Basic Issues in Hepatology" was commissioned by the editorial office without any funding or sponsorship. JU reports personal fees from Allergan, personal fees from AveXis, personal fees from Astra Zeneca, personal fees from CSL, personal fees from Cytogel, grants from LB Pharmaceuticals, personal fees from SK Life Sciences, outside the submitted work. The other author has no other conflicts of interest to declare.

Ethical Statement: The authors are accountable for all aspects of the work in ensuring that questions related to the accuracy or integrity of any part of the work are appropriately investigated and resolved.

Open Access Statement: This is an Open Access article 
distributed in accordance with the Creative Commons Attribution-NonCommercial-NoDerivs 4.0 International License (CC BY-NC-ND 4.0), which permits the noncommercial replication and distribution of the article with the strict proviso that no changes or edits are made and the original work is properly cited (including links to both the formal publication through the relevant DOI and the license). See: https://creativecommons.org/licenses/by-nc-nd/4.0/.

\section{References}

1. Gao B, Xu MJ, Bertola A, et al. Animal models of alcoholic liver disease: pathogenesis and clinical relevance. Gene Expr 2017;17:173-86.

2. Neuman MG, French SW, Zakhari S, et al. Alcohol, microbiome, life style influence alcohol and non-alcoholic organ damage. Exp Mol Pathol 2017;102:162-80.

3. Perwaiz S, Forrest D, Mignault D, et al. Appearance of atypical 3 alpha, 6 beta, 7 beta, 12 alpha-tetrahydroxy- 5 beta-cholan-24-oic acid in spgp knockout mice. J Lipid Res 2003;44:494-502.

4. Teschke R. Alcoholic liver disease: alcohol metabolism, cascade of molecular mechanisms, cellular targets, and clinical aspects. Biomedicines 2018;6:106.

5. Teschke R. Alcoholic liver disease: current mechanistic aspects with focus on their clinical relevance. Biomedicines 2019;7:68.

6. Hasumura Y, Teschke R, Lieber CS. Increased carbon tetrachloride hepatotoxicity, and its mechanism, after chronic ethanol consum.ption. Gastroenterology 1974;66:415-22.

7. Scholten D, Trebicka J, Liedtke C, et al. The carbon tetrachloride model in mice. Lab Anim 2015;49:4-11.

8. Teschke R. Aliphatic halogenated hydrocarbons: report and analysis of liver injury in 60 patients. J Clin Transl Hepatol 2018;6:350-61.

9. Teschke R. Liver injury by carbon tetrachloride intoxication in 16 patients treated with forced ventilation to accelerate toxin removal via the lungs: A clinical report. Toxics 2018;6:25.

10. Weiskirchen R, Lammert F. A yellow bullet against the drivers of hepatic fibrogenesis. Hepatology 2008;48:683-5.

11. Weiskirchen R, Meurer SK, Liedtke C, et al. Mast cells in liver fibrogenesis. Cells 2019;8:1429.

12. Gehrke SG, Kulaksiz H, Herrmann T, et al. Expression of hepcidin in hereditary hemochromatosis: evidence for a regulation in response to the serum transferrin saturation and to non-transferrin-bound iron. Blood 2003;102:371-6.
13. Niederau C, Fischer R, Pürschel A, et al. Long-term survival in patients with hereditary hemochromatosis. Gastroenterology 1996;110:1107-19.

14. Merle U, Schaefer M, Ferenci P, et al. Clinical presentation, diagnosis and long-term outcome of Wilson's disease: a cohort study. Gut 2007;56:115-20.

15. Stremmel W, Merle U, Weiskirchen R. Clinical features of Wilson disease. Ann Transl Med 2019;7:S61.

16. Jing J, Teschke R. Traditional Chinese Medicine and herbinduced liver injury: comparison with drug-induced liver injury. J Clin Transl Hepatol 2018;6:57-68.

17. Shahbaz O, Mahajan S, Lewis JH. Highlights of drug- and herb- induced liver injury in the literature from 2016: how best to translate new information into clinical practice. Expert Opin Drug Metab Toxicol 2017;13:935-51.

18. Teschke R, Zhu Y, Jing J. Herb-induced liver injury in Asia: Current role of RUCAM for causality assessment in 11,160 published cases of HILI. J Clin Transl Hepatol 2020;8:200-14.

19. Zhu Y, Niu M, Chen J, et al. Comparison between Chinese herbal medicine and Western medicine-induced liver injury of 1985 patients. J Gastroenterol Hepatol 2016;31:1476-1482.

20. Real M, Barnhill MS, Higley C, et al. Drug-induced liver injury: highlights of the recent literature. Drug Saf 2019;42:365-87.

21. Sarges P, Steinberg JM, Lewis JH. Drug-Induced Liver Injury: Highlights from a review of the 2015 literature. Drug Saf 2016;39:801-21.

22. Teschke R. Top-ranking drugs out of 3312 drug-induced liver injury cases evaluated by the Roussel Uclaf Causality Assessment Method. Expert Opin Drug Metab Toxicol 2018;14:1169-87.

23. Teschke R. Analysis of 46,266 cases assessed for causality by RUCAM and published from 2014 to early 2019. In: Special issue: Clinical drug induced liver injury: Current diagnostic and mechanistic challenges, guest editors: Rolf Teschke, Gaby Danan, James H. Lewis. Front Pharmacol 2019;10:730.

24. Uetrecht J. Mechanistic studies of idiosyncratic DILI: Clinical implications. Front Pharmacol 2019;10:837.

25. Björnsson ES, Hoofnagle JH. Categorization of drugs implicated in causing liver injury: Critical assessment based on published case reports. Hepatology 2016;63:590-603.

26. Björnsson ES. Hepatotoxicity by drugs: The most common implicated agents. Int J Mol Sci 2016;17:224.

27. Shi Q, Hong H, Senior J, et al. Biomarkers for druginduced liver injury. Expert Rev Gastroenterol Hepatol 2010;4:225-34. 
28. Teschke R, Frenzel C, Wolff A, et al. Drug induced liver injury: accuracy of diagnosis in published reports. Ann Hepatol 2014;13:248-55.

29. Teschke R, Danan G. Drug induced liver injury with analysis of alternative causes as confounding variables. $\mathrm{Br} \mathrm{J}$ Clin Pharmacol 2018;84:1467-77.

30. Thakkar S, Chen M, Hong H, et al. Drug-induced liver injury (DILI) classification and its application on human DILI risk prediction. In: Chen M, Will Y. editors. DrugInduced Liver Toxicity. Springer, 2018:45-59.

31. Teschke R, Danan G. Causality assessment methods in drug-induced liver injury. In: Chen M, Will Y. editors. Drug-induced liver toxicity. Springer, 2018:555-94.

32. Noureddin N, Kaplowitz N. Overview of mechanisms of drug-induced liver injury (DILI) and key challenges in DILI research. In: Chen M, Will Y. editors. Drug-Induced Liver Toxicity. Springer, 2018:3-18.

33. Danan G, Benichou C. Causality assessment of adverse reactions to drugs--I. A novel method based on the conclusions of international consensus meetings: application to drug-induced liver injuries. J Clin Epidemiol 1993;46:1323-30.

34. Danan G, Teschke R. RUCAM in drug and herb induced liver injury: the update. nt J Mol Sci 2016;17:14.

35. Aithal GP, Rawlins MD, Day CP. Accuracy of hepatic adverse drug reaction reporting in one English health region. BMJ 1999;319:1541.

36. Teschke R, Eickhoff A, Brown AC, et al. Diagnostic biomarkers in liver injury by drugs, herbs, and alcohol: tricky dilemma after EMA correctly and officially retracted Letter of Support. Int J Mol Sci 2019;21:212.

37. EMA. Retraction of the "Letter of support for drug-induced liver injury (DILI) biomarker" (EMA/423870/2016). Available online: https:// www.europa.eu.documents/other/retraction-lettersupport-drug-induced-liver-injury-dili-biomarkerema/423870/2016_en.pdf. Last accessed on 15 April 2019.

38. Kleiner DE. Recent advances in the histopathology of druginduced liver injury. Surg Pathol Clin 2018;11:297-311.

39. Morán L, Cubero FJ. Extracellular vesicles in liver disease and beyond. World J Gastroenterol 2018;24:4519-26.

40. Urban SK, Mocan T, Sänger H, et al. Extracellular vesicles in liver diseases: diagnostic, prognostic, and therapeutic application. Semin Liver Dis 2019;39:70-7.

41. Bala S, Petrasek J, Mundkur S, et al. Circulating microRNAs in exosomes indicate hepatocyte injury and inflammation in alcoholic, drug-induced, and inflammatory liver diseases. Hepatology 2012;56:1946-57.
42. Cho YE, Mezey E, Hardwick JP, et al. Increased ethanolinducible cytochrome P450-2E1 and cytochrome P450 isoforms in exosomes of alcohol-exposed rodents and patients with alcoholism through oxidative and endoplasmic reticulum stress. Hepatol Commun 2017;1:675-90.

43. Gerth K, Kodidela S, Mahon M, et al. Circulating extracellular vesicles containing xenobiotic metabolizing CYP enzymes and their potential roles in extrahepatic cells via cell-cell interactions. Int J Mol Sci 2019;20:6178.

44. Gloor Y, Schvartz D, F Samer C. Old problem, new solutions: biomarker discovery for acetaminophen liver toxicity. Expert Opin Drug Metab Toxicol 2019;15:659-69.

45. Holman NS, Mosedale M, Wolf KK, et al. Subtoxic alterations in hepatocyte-derived exosomes: an early step in drug-induced liver injury. Toxicol Sci 2016;151:365-75.

46. Jeppesen DK, Fenix AM, Franklin JL, et al. Reassessment of exosome composition. Cell 2019;177:428-445.e18.

47. Masyuk AI, Masyuk TV, Larusso NF. Exosomes in the pathogenesis, diagnostics and therapeutics of liver diseases. J Hepatol 2013;59:621-5.

48. Ogese MO, Jenkins RE, Adair K, et al. Exosomal transport of hepatocyte-derived drug-modified proteins to the immune system. Hepatology 2019;70:1732-49.

49. Rahman MA, Kodidela S, Sinha N, et al. Plasma exosomes exacerbate alcohol- and acetaminophen-induced toxicity via CYP2E1 pathway. Sci Rep 2019;9:6571.

50. Seo W, Eun HS, Kim SY, et al. Exosome-mediated activation of toll-like receptor 3 in stellate cells stimulates interleukin-17 production by $\gamma \delta \mathrm{T}$ cells in liver fibrosis. Hepatology 2016;64:616-31.

51. Teschke R. Microsomal ethanol-oxidizing system: success over 50 years and an encouraging future. Alcohol Clin Exp Res 2019;43:386-400.

52. Starkey Lewis PJ, Dear J, Platt V, et al. Circulating microRNAs as potential markers of human drug-induced liver injury. Hepatology 2011;54:1767-76.

53. Cho YE, Kim SH, Lee BH, et al. Circulating plasma and exosomal micrornas as indicators of drug-induced organ injury in rodent models. Biomol Ther (Seoul) 2017;25:367-73.

54. Church RJ, Watkins PB. Serum biomarkers of druginduced liver injury: Current status and future directions. J Dig Dis 2019;20:2-10.

55. Enache LS, Enache EL, Ramière C, et al. Circulating RNA molecules as biomarkers in liver disease. Int J Mol Sci 2014;15:17644-66.

56. Fontana RJ. Pathogenesis of idiosyncratic drug-induced liver injury and clinical perspectives. Gastroenterology 2014;146:914-28. 
57. Krauskopf J, Kleinjans J, de Kok TM. Circulating microRNAs as novel biomarkers of drug-induced liver injury in humans. In: Chen M, Will Y, editors. DrugInduced Liver Toxicity. Springer, 2018:597-610.

58. Li LM, Wang D, Zen K. MicroRNAs in drug-induced liver injury. J Clin Transl Hepatol 2014;2:162-9.

59. McGill MR, Jaeschke H. MicroRNAs as signaling mediators and biomarkers of drug- and chemical-induced liver injury. J Clin Med 2015;4:1063-78.

60. Thulin P, Nordahl G, Gry M, et al. Keratin-18 and microRNA-122 complement alanine aminotransferase as novel safety biomarkers for drug-induced liver injury in two human cohorts. Liver Int 2014;34:367-78.

61. Antoine D. Translational and mechanistic biomarkers of drug-induced liver injury - Candidates and qualification strategies. In: Chen M, Will Y. editors. Drug-Induced Liver Toxicity. Springer, 2018:533-53.

62. Senior JR. New biomarkers for drug-induced liver injury: are they really better? What do they diagnose. Liver Int 2014;34:325-7.

63. Teschke R, Schulze J, Eickhoff A, et al. Drug induced liver injury: can biomarkers assist RUCAM in causality assessment. Int J Mol Sci 2017;18:803.

64. Vliegenthart ADB, Berends C, Potter CMJ, et al. MicroRNA-122 can be measured in capillary blood which facilitates point-of-care testing for drug-induced liver injury. Br J Clin Pharmacol 2017;83:2027-33.

65. Konturek PC, Harsch IA, Konturek K, et al. Gut'liver axis: How do gut bacteria influence the liver. Med Sci (Basel) 2018;6:79.

66. Dubin K, Callahan MK, Ren B, et al. Intestinal microbiome analyses identify melanoma patients at risk for checkpoint-blockade-induced colitis. Nat Commun 2016;7:10391.

67. Cani PD. Human gut microbiome: hopes, threats and promises. Gut 2018;67:1716-25.

68. Nolan JP. The role of intestinal endotoxin in liver injury: a long and evolving history. Hepatology 2010;52:1829-35.

69. Zhang J, Wang R. Gut microbiota modulates drug pharmacokinetics. Drug Metab Rev 2018;50:357-68.

70. Borkham-Kamphorst E, Van de Leur E, Haas U, et al. Liver parenchymal cells lacking Lipocalin 2 (LCN2) are prone to endoplasmic reticulum stress and unfolded protein response. Cell Signal 2019;55:90-9.

71. Su GL, Gong KQ, Fan MH, et al. Lipopolysaccharidebinding protein modulates acetaminophen-induced liver injury in mice. Hepatology 2005;41:187-95.

72. Teschke R, Zhu Y. Opinion: Intestinal microbiome, endotoxins, cytochrome P450 2E1, and the gut-liver axis in alcoholic liver disease. EC Gastroenterology Dig Syst 2019;6:66-75.

73. Tacke F. Targeting hepatic macrophages to treat liver diseases. J Hepatol 2017;66:1300-12.

74. Dragoi D, Benesic A, Pichler G, et al. Proteomics analysis of monocyte-derived hepatocyte-like cells identifies Integrin Beta 3 as a specific biomarker for drug-induced liver injury by diclofenac. Front Pharmacol 2018;9:699.

75. Iorga A, Dara L, Kaplowitz N. Drug-Induced Liver Injury: Cascade of events leading to cell death, apoptosis or necrosis. In special issue: Molecular Research on Drug Induced liver Injury, academic editors: Rolf Teschke and Gaby Danan. Int J Mol Sci 2017;18:1018.

76. Uetrecht J. Immunoallergic drug-induced liver injury in humans. Semin Liver Dis 2009;29:383-92.

77. Uetrecht J, Naisbitt DJ. Idiosyncratic adverse drug reactions: current concepts. Pharmacol Rev 2013;65:779-808.

78. Tacke F, Zimmermann HW. Macrophage heterogeneity in liver injury and fibrosis. J Hepatol 2014;60:1090-6.

79. Kalra BS. Cytochrome P450 enzyme isoforms and their therapeutic implications: an update. Indian J Med Sci 2007;61:102-16.

80. Sychev DA, Ashraf GM, Svistunov AA, et al. The cytochrome $\mathrm{P} 450$ isoenzyme and some new opportunities for the prediction of negative drug interaction in vivo. Drug Des Devel Ther 2018;12:1147-56.

81. Foti RS, Dalvie DK. Cytochrome P450 and noncytochrome $\mathrm{P} 450$ oxidative metabolism: contributions to the pharmacokinetics, safety, and efficacy of xenobiotics. Drug Metab Dispos 2016;44:1229-45.

82. Regan SL, Maggs JL, Hammond TG, et al. Acyl glucuronides: the good, the bad and the ugly. Biopharm Drug Dispos 2010;31:367-95.

83. Acocella G. Pharmacokinetics and metabolism of rifampin in humans. Rev Infect Dis 1983;5 Suppl 3:S428-32.

84. Amano T, Fukami T, Ogiso T, et al. Identification of enzymes responsible for dantrolene metabolism in the human liver: A clue to uncover the cause of liver injury. Biochem Pharmacol 2018;151:69-78.

85. Andrade C, Freitas L, Oliveira V. Twenty-six years of HIV science: an overview of anti-HIV drugs metabolism. Braz J Pharmaceut Sci 2011;47:209-30.

86. Back DJ, Tjia JF, Karbwang J, et al. In vitro inhibition studies of tolbutamide hydroxylase activity of human liver microsomes by azoles, sulphonamides and quinolines. Br J Clin Pharmacol 1988;26:23-9.

87. Bertz RJ, Granneman GR. Use of in vitro and in vivo data 
to estimate the likelihood of metabolic pharmacokinetic interactions. Clin Pharmacokinet 1997;32:210-58.

88. Björnsson E, Olsson R. Outcome and prognostic markers in severe drug-induced liver disease. Hepatology 2005;42:481-9.

89. Choughule KV, Barnaba C, Joswig-Jones CA, et al. In vitro oxidative metabolism of 6-mercaptopurine in human liver: insights into the role of the molybdoflavoenzymes aldehyde oxidase, xanthine oxidase, and xanthine dehydrogenase. Drug Metab Dispos 2014;42:1334-40.

90. Das KM, Chowdhury JR, Zapp B, et al. Small bowel absorption of sulfasalazine and its hepatic metabolism in human beings, cats, and rats. Gastroenterology 1979;77:280-4.

91. Dekker SJ, Dohmen F, Vermeulen NPE, et al. Characterization of kinetics of human cytochrome P450s involved in bioactivation of flucloxacillin: inhibition of CYP3A-catalysed hydroxylation by sulfaphenazole. Br J Pharmacol 2019;176:466-77.

92. Desta Z, Saussele T, Ward B, et al. Impact of CYP2B6 polymorphism on hepatic efavirenz metabolism in vitro. Pharmacogenomics 2007;8:547-58.

93. Donehower RC. Metabolic conversion of methotrexate in man. Recent results. Cancer Res 1980;74:37-41.

94. Dybing E, Nelson SD, Mitchell JR, et al. Oxidation of alpha-methyldopa and other catechols by cytochrome P-450-generated superoxide anion: possible mechanism of methyldopa hepatitis. Mol Pharmacol 1976;12:911-20.

95. Erickson DA, Mather G, Trager WF, et al. Characterization of the in vitro biotransformation of the $\mathrm{HIV}-1$ reverse transcriptase inhibitor nevirapine by human hepatic cytochromes P-450. Drug Metab Dispos 1999;27:1488-95.

96. Fatunde OA, Brown SA. The role of CYP450 Drug metabolism in precision cardio-oncology. Int J Mol Sci 2020;21:604.

97. Hautekeete ML, Horsmans Y, Van Waeyenberge C, et al. HLA association of amoxicillin-clavulanate--induced hepatitis. Gastroenterology 1999;117:1181-6.

98. Heidari R, Niknahad H, Jamshidzadeh A, et al. An overview on the proposed mechanisms of antithyroid drugs-induced liver injury. Adv Pharm Bull 2015;5:1-11.

99. Hopley L, van Schalkwyk J. Cytochrome P450 - Just the basics. Available online: https://www.anaesthetist.com/ physiol/basics/metabol/cyp/Findex.htm-cyp.htm. Accessed 15 April 2020.

100.Johansson I, Ingelman-Sundberg M. Genetic polymorphism and toxicology - with emphasis on cytochrome p450. Toxicol Sci 2011;120:1-13.

101. Kiang TK, Ho PC, Anari MR, et al. Contribution of CYP2C9, CYP2A6, and CYP2B6 to valproic acid metabolism in hepatic microsomes from individuals with the CYP2C9*1/*1 genotype. Toxicol Sci 2006;94:261-71.

102. Kim JH, Choi WG, Lee S, et al. Revisiting the metabolism and bioactivation of ketoconazole in human and mouse using liquid chromatography-mass spectrometry-based metabolomics. Int J Mol Sci 2017;18:621.

103.Landowski CP, Song X, Lorenzi PL, et al. Floxuridine amino acid ester prodrugs: enhancing Caco-2 permeability and resistance to glycosidic bond metabolism. Pharm Res 2005;22:1510-8.

104. LiverTox. Clinical and research information on drug-induced liver injury. Bethesda (MD): National Institute of Diabetes and Digestive and Kidney Diseases; 2012. infliximab. Last updated 10 February 2017. Available online: https//www.nbci. nim.nih.gov/books/. Accessed 15 April 2020.

105. Myers AL, Kawedia JD, Champlin RE, et al. Clarifying busulfan metabolism and drug interactions to support new therapeutic drug monitoring strategies: a comprehensive review. Expert Opin Drug Metab Toxicol 2017;13:901-23.

106. Nelis HJ, De Leenheer AP. Metabolism of minocycline in humans. Drug Metab Dispos 1982;10:142-6.

107. Okuno H, Kitao Y, Takasu M, et al. Depression of drug metabolizing activity in the human liver by interferonalpha. Eur J Clin Pharmacol 1990;39:365-7.

108. Scott LM, Durant P, Leone-Kabler S, et al. Effects of prior oral contraceptive use and soy isoflavonoids on estrogenmetabolizing cytochrome P450 enzymes. J Steroid Biochem Mol Biol 2008;112:179-85.

109. Shi J, Montay G, Bhargava VO. Clinical pharmacokinetics of telithromycin, the first ketolide antibacterial. Clin Pharmacokinet 2005;44:915-34.

110. Shih TY, Pai CY, Yang P, et al. A novel mechanism underlies the hepatotoxicity of pyrazinamide. Antimicrob Agents Chemother 2013;57:1685-90.

111. Talseth T. Kinetics of hydralazine elimination. Clin Pharmacol Ther 1977;21:715-20.

112. Turnheim K, Krivanek P, Oberbauer R. Pharmacokinetics and pharmacodynamics of allopurinol in elderly and young subjects. Br J Clin Pharmacol 1999;48:501-9.

113. Wang Y, Gray JP, Mishin V, et al. Role of cytochrome $\mathrm{P} 450$ reductase in nitrofurantoin-induced redox cycling and cytotoxicity. Free Radic Biol Med 2008;44:1169-79.

114. Yamazaki H, Shimada T. Progesterone and testosterone hydroxylation by cytochromes P450 2C19, 2C9, and $3 \mathrm{~A} 4$ in human liver microsomes. Arch Biochem Biophys 
1997;346:161-9.

115. Yu K, Geng X, Chen M, et al. High daily dose and being a substrate of cytochrome P450 enzymes are two important predictors of drug-induced liver injury. Drug Metab Dispos 2014;42:744-50.

116.Zanger UM, Schwab M. Cytochrome P450 enzymes in drug metabolism: regulation of gene expression, enzyme activities, and impact of genetic variation. Pharmacol Ther 2013;138:103-41.

117. Brunell D, Sagher D, Kesaraju S, et al. Studies on the metabolism and biological activity of the epimers of sulindac. Drug Metab Dispos 2011;39:1014-21.

118. Nielsen TL, Rasmussen BB, Flinois JP, et al. In vitro metabolism of quinidine: the (3S)-3-hydroxylation of quinidine is a specific marker reaction for cytochrome P-4503A4 activity in human liver microsomes. J Pharmacol Exp Ther 1999;289:31-7.

119. Guengerich FP. Mechanisms of cytochrome P450 substrate oxidation: MiniReview. J Biochem Mol Toxicol 2007;21:163-8.

120.Fu S, Wu D, Jiang W, et al. Molecular biomarkers in druginduced liver injury: challenges and future perspectives. Front Pharmacol 2020;10:1667.

121. Uetrecht JP. New concepts in immunology relevant to idiosyncratic drug reactions: the "danger hypothesis" and innate immune system. Chem Res Toxicol 1999;12:387-95.

122. Kumar S. Plasma exosomes and drug metabolic cytochrome p450 enzymes. J Drug Metab Toxicol 2015;6:5671.

123. Kumar S, Sinha N, Gerth KA, et al. Specific packaging and circulation of cytochromes $\mathrm{P} 450$, especially $2 \mathrm{E} 1$ isozyme, in human plasma exosomes and their implications in cellular communications. Biochem Biophys Res Commun 2017;491:675-80.

124. Metushi IG, Sanders C, Acute LSG, et al. Detection of anti-isoniazid and anti-cytochrome $\mathrm{P} 450$ antibodies in patients with isoniazid-induced liver failure. Hepatology 2014;59:1084-93.

125. Obermayer-Straub P, Strassburg CP, Manns MP. Target proteins in human autoimmunity: cytochromes $\mathrm{P} 450$ and UDP-glucuronosyltransferases. Can J Gastroenterol 2000;14:429-39.

126. Sutti S, Rigamonti C, Vidali M, et al. CYP2E1 autoantibodies in liver diseases. Redox Biol 2014;3:72-8.

127. Mosedale M, Watkins PB. Drug-induced liver injury: Advances in mechanistic understanding that will inform risk management. Clin Pharmacol Ther 2017;101:469-80. 128. Boelsterli UA, Lee KK. Mechanisms of isoniazid-induced idiosyncratic liver injury: emerging role of mitochondrial stress. J Gastroenterol Hepatol 2014;29:678-87.

129. Kaplowitz N. Avoiding idiosyncratic DILI: two is better than one. Hepatology 2013;58:15-7.

130. Ye H, Nelson LJ, Gómez Del Moral M, et al. Dissecting the molecular pathophysiology of drug-induced liver injury. World J Gastroenterol 2018;24:1373-85.

131.Argikar UA, Potter PM, Hutzler JM, et al. Challenges and opportunities with non-CYP enzymes aldehyde oxidase, carboxylesterase, and UDP-glucuronosyltransferase: Focus on reaction phenotyping and prediction of human clearance. AAPS J 2016;18:1391-405.

132. Boelsterli UA. Acyl glucuronides: mechanistic role in drug toxicity. Curr Drug Metab 2011;12:213-4.

133. Shah P, Sundaram V, Björnsson E. Biologic and checkpoint inhibitor-induced liver injury: a systematic literature review. Hepatol Commun 2020;4:172-84.

134.McGill MR, Jaeschke H. Animal models of druginduced liver injury. Biochim Biophys Acta Mol Basis Dis 2019;1865:1031-9.

135.Pan Y, Cao M, You D, et al. Research progress on the animal models of drug-induced liver injury: current status and further perspectives. Biomed Res Int 2019;2019:1283824.

136. Ghahremanloo A, Soltani A, Modaresi SMS, et al. Recent advances in the clinical development of immune checkpoint blockade therapy. Cell Oncol (Dordr) 2019;42:609-26.

137. Suzman DL, Pelosof L, Rosenberg A, et al. Hepatotoxicity of immune checkpoint inhibitors: An evolving picture of risk associated with a vital class of immunotherapy agents. Liver Int 2018;38:976-87.

138. Metushi IG, Hayes MA, Uetrecht J. Treatment of PD1(-/-) mice with amodiaquine and anti-CTLA4 leads to liver injury similar to idiosyncratic liver injury in patients. Hepatology 2015;61:1332-42.

139. Mak A, Uetrecht J. The Role of CD8 T cells in amodiaquine-induced liver injury in PD1-/- Mice cotreated with anti-CTLA-4. Chem Res Toxicol 2015;28:1567-73.

140. Mak A, Uetrecht J. The combination of anti-CTLA-4 and PD1-/- mice unmasks the potential of isoniazid and nevirapine to cause liver injury. Chem Res Toxicol 2015;28:2287-91.

Cite this article as: Teschke R, Uetrecht $\mathrm{J}$. Mechanism of idiosyncratic drug induced liver injury (DILI): unresolved basic issues. Ann Transl Med 2021;9(8):730. doi: 10.21037/atm-2020ubih-05 